\title{
Atendimento e gestão como indicador de qualidade no serviço de emergência: revisão de literatura
}

\author{
Attention and management as an indicator of quality in the emergency \\ service: literature review
}

José Nazareth Barbosa Santos Filho ${ }^{1}$, Isabella Froes Sousa ${ }^{2}$, Stefane e Silva Lima ${ }^{2}$, Nailde Melo Santos ${ }^{3}$, Daniela Bassi ${ }^{4}$, Ilana Almeida Felipe ${ }^{4}$

Resumo: O acolhimento com classificação de risco é a ferramenta preconizada pelo Ministério da Saúde nos serviços de emergências para facilitar o tempo de atendimentos e as prioridades de acordo com o grau de gravidade de cada situação. Assim, a avaliação e o monitoramento da qualidade dos serviços de acolhimento na urgência e emergência são indispensáveis na melhoria do atendimento ao usuário que procura o serviço hospitalar de emergência. A revisão incluiu estudos do tipo artigos e periódicos publicados entre 2013 e 2017, indexados nas bases de dados do Literatura Latino-americana e do Caribe em Ciências da Saúde (LILACS), Scientific Eletronic Library Online (SCIELO) e Biblioteca Virtual em Saúde (BVS). Os resultados demonstram uma nova realidade de ambientes e parâmetros de trabalho, identificando aspectos de condições inadequadas e ausência de prioridades em atendimentos graves. Destacando a gestão do cuidado por equipes multiprofissionais, ampliando responsabilidades. Dentre os principais enfoques abordados na realidade encontrada, é de suma importância a identificação de necessidades da estrutura física e de capacidade profissional, priorizando o usuário dentro de uma gestão com qualidade no atendimento.

Palavras chaves: Gestão da Qualidade; Indicadores de Qualidade em Assistência à Saúde; Atendimento de Emergência.

\begin{abstract}
The host with risk classification is the tool recommended by the Ministry of Health in the emergency services to facilitate the time of care and the priorities according to the level of severity of each situation. Thus, the evaluation and monitoring of the quality of the reception services in the urgency and emergency are indispensable in the improvement of the service to the user who seeks the hospital emergency service. The review included studies of articles and journals published between 2013 and 2017, indexed in the Literatura Latino-Americana e do Caribe em Ciências da Saúde (LILACS), Scientific Electronic Library Online (SCIELO) and Biblioteca Virtual em Saúde (BVS). The results demonstrate a new reality of environments and work parameters, identifying aspects of inadequate conditions and lack of priorities in serious care. Highlighting the management of care by multiprofessional teams, increasing responsibilities. Among the main approaches addressed in the reality found, it is of utmost importance to identify the needs of the physical structure and professional capacity, prioritizing the user within a management with quality in the service.
\end{abstract}

Keywords: Quality Management; Quality Indicators in Health Care; Emergency Care.

\footnotetext{
Docente do Curso de Medicina da Universidade Ceuma.

Alunas do Curso de Graduação em Medicina da Universidade Ceuma.

Enfermeira da Universidade Federal do Maranhão.

4 Docentes do Programa de Pós-Graduação de Gestão de Programas e Serviços de Saúde da Universidade Ceuma.
}

Autor correspondente: José Nazareth Barbosa Santos Filho. Rua Balsas, Quadra 46, Casa 3, Bairro Quintas do Calhau, São Luís-MA. CEP: 65072-011. Contato: (98) 98116-4430. E-mail: jnbsf@terra.com.br. 
Introdução

As mudanças que estão ocorrendo no setor da saúde e no padrão de comportamento da sociedade, levam a necessidade de melhoria na qualidade de forma geral $^{1}$. As pessoas que buscam atendimento querem mais qualidade, uma vez que conhecendo, exigem os seus direitos.

O destaque atribuído a qualidade em saúde toma força nos serviços particulares, mas vai gradativamente surgindo e se destacando também no serviço público, com $\mathrm{o}$ advento $\mathrm{e}$ conhecimento dos direitos sociais e dos direitos dos consumidores. No serviço hospitalar de uma forma geral, e em especial no serviço de urgência e emergência, vários acontecimentos contribuíram para a melhoria e a necessidade de investir em qualidade ${ }^{2}$.

Caracterizado como um fenômeno mundial, o movimento pela qualidade nos serviços de saúde visa atender à crescente exigência dos usuários por melhorias no atendimento, fato que tem suscitado numerosas discussões e investigações ${ }^{3}$.

Os mecanismos de avaliação da qualidade dos serviços de saúde no ambiente hospitalar, existem há muito tempo, porém, mais do que nunca, o tema vem ganhando espaço nas discussões do mundo inteiro. Os elevados custos da assistência, ao aumento dos processos contra os erros médicos e à exigência dos usuários por um serviço de qualidade, a Avaliação da qualidade passou a ser um tema prioritário nessa época 4 .
No Brasil o primeiro trabalho desenvolvido para melhorar a qualidade dos serviços foi a utilização da Ficha de Inquérito Hospitalar, proposta pelo médico Odair Pedroso em 1935, a qual especificava como deveria ser a organização de uma instituição hospitalar naquela época ${ }^{5}$.

Após este período, surge a implantação de um sistema de acompanhamento da qualidade dos serviços de saúde é recente, tendo sido iniciada a partir dos anos 1990, quando as instituições públicas e privadas no país passaram a desenvolver programas para a garantia da qualidade ${ }^{6}$.

Portanto, espera-se que as instituições para garantir qualidade devam ter área física, recursos humanos, materiais, financeiros e modelo organizacional, bem como relações entre prestador e receptor dos cuidados e clareza das características desejáveis dos produtos ${ }^{7}$.

A porta de emergência hospitalar objetiva classificar e priorizar os atendimentos diminuindo assim as morbidades, mortalidades e as sequelas decorrentes da demora do atendimento e que possam incapacitar as pessoas. Deve-se incluir uma gestão que contemple os recursos necessários no âmbito humano, material, infraestrutura e equipamentos, garantindo qualidade da assistência de forma integral e contínua ${ }^{8}$.

O crescente número de acidentes, a violência urbana e a estrutura hospitalar de atendimento considerada inadequada na rede de serviços de saúde, são fatores preponderantes na sobrecarga dos serviços. O conhecimento desta 
realidade em nosso meio é de importância fundamental, no sentido de evidenciar a necessidade de reestruturação do atual sistema de saúde, na perspectiva de consolidação dos princípios do SUS ${ }^{9}$.

Embora o processo de trabalho nas instituições de saúde tenha passado por transformações que trouxeram avanços científicos e tecnológicos, ainda são vivenciados atendimentos desumanos, filas de espera, índices elevados de infecção hospitalar, gerenciamento ineficaz de custos, baixo nível de capacitação profissional e ausência de educação continuada/permanente,

dimensionamento de pessoal inadequado, entre outras dificuldades. Essa situação clama por modelos de gestão adequados, que otimizem os recursos aplicados, melhorando a produtividade, a satisfação dos usuários e dos profissionais que atuam nos serviços de saúde ${ }^{10}$.

Segundo o Ministério da Saúde, dentre os setores/serviços de um hospital, é provável que o Serviço Hospitalar de Emergência (SHE) seja um dos mais críticos em relação à promoção da qualidade no atendimento, porque de acordo com - Ministério da Saúde é nesse ambiente que se observa, entre outros problemas, a falta de hierarquia no atendimento aos agravos e a desordem nos fluxos internos que são gerados pela elevada procura de usuários por atendimento ${ }^{11}$.

Este artigo visa responder aos questionamentos: Qual a produção científica sobre os indicadores de qualidade na assistência à saúde na unidade de emergência? Em função da produção científica escassa sobre a gestão da emergência, incluindo no aspecto gerencial que reflete na estrutura física, na assistência direta aos pacientes e na qualidade de trabalho dos profissionais.

Sintetizando a produção cientifica nacional e internacional sobre a qualidade dos serviços de emergência no período de 2013 a 2017. A emergência é considerada uma situação de preocupação para os gestores e profissionais em função da gravidade dos pacientes atendidos ou ainda que simplesmente procuram atendimento.

\section{Método}

Trata-se de um estudo de revisão integrativa da literatura sobre Classificação de risco hospitalar como indicador de qualidade no serviço de emergência. A revisão incluiu estudos do tipo artigos e periódicos publicados entre 2013 e 2017, indexados nas bases de dados do Literatura Latino-americana e do Caribe em Ciências da Saúde (LILACS), Scientific Eletronic Library Online (SCIELO) e Biblioteca Virtual em Saúde (BVS).

A realização desse estudo partiu da seleção dos artigos que fizeram parte das buscas, utilizando-se os descritores: Gestão da Qualidade; Indicadores de Qualidade em Assistência à Saúde; Atendimento de Emergência. Os critérios de inclusão foram estudos que incluíram serviço hospitalar em pacientes adultos na urgência e emergência com indicações de classificação de risco, utilizando a 
porta de emergência como primeiro atendimento. Poderiam ser estudos descritivos, revisão de literatura, série de casos, estudos tipo antes e depois, em língua inglesa e portuguesa

Durante as buscas de dados foram utilizadas as combinações entre os descritores do DECS de modo a encontrar 232 artigos relacionados ao assunto e após a análise selecionados 09 que se adequaram ao objeto do estudo. Após a leitura dos títulos e resumos, foram incluídos para essa análise os artigos/estudos que atenderam aos critérios de elegibilidade, possibilitando conhecer os aspectos sobre Gestão da qualidade e indicadores no serviço de emergência.

A análise ocorreu a pós a seleção das publicações e da coleta dos dados de interesse incluindo: autores, ano de publicação, periódico e objetivo do estudo publicado. $\mathrm{Na}$ primeira etapa os dados de localização, ano de publicação e o periódico forma analisados, e após foi feita uma segunda etapa dos artigos a partir dos objetivos e resultados apresentados, inferindo os dados em categorias por similaridades de assuntos.

\section{Resultados e discussão}

Foram encontrados 232 artigos e, após análise primária, selecionou-se apenas 09 artigos que atenderam aos critérios de inclusão previamente estabelecidos. As publicações encontradas no período de 2013 e 2017 e que estavam relacionadas ao tema (quadro 1).
A localização dos estudos na íntegra foi realizada em acesso à biblioteca virtual no ambiente da PUBMED, com buscas utilizando os descritores e os respectivos cruzamentos destes.

Realizada a leitura detalhada dos 09 artigos encontrados, o que permitiu agrupar os resultados em categorias por assuntos abordados, constituindo assim, quatro categorias distribuídas como seguem: Indicadores de qualidade em assistência à saúde (23\%); Avaliação dos serviços de saúde (37\%), Atendimento de Emergência (19\%) e Qualidade da assistência (21\%).

Quanto ao tipo de estudo, foram encontrados vários tipos de abordagens: Estudo retrospectivo; Revisão sistemática da literatura; Estudo transversal; Pesquisa descritiva-exploratória; Revisão integrativa; Pesquisa de campo; Relato de experiência e Pesquisa qualitativa.

Os estudos analisados envolvendo os indicadores de qualidade em assistência à saúde apontam para uma realidade de novos ambientes e parâmetros do processo de trabalho, envolvendo a opinião dos profissionais nos serviços de emergência. Deslane em seu estudo, ressalta duas características do processo de trabalho. A primeira é a responsabilização e vínculo das equipes, alternando entre 0 heroísmo e o descaso, distinguindose por: a) esgotamento físico e mental dos profissionais; b) abandono da evolução clínica dos pacientes aguardando vaga para internar; c) inobservância dos direitos dos pacientes; d) violação dos princípios éticos profissionais; 
Quadro 1 - Distribuição e análise dos estudos sobre Atendimento e gestão como indicador de qualidade no serviço de emergência de 2013 a 2017

\begin{tabular}{|c|c|c|c|}
\hline Título & Objetivo do estudo & Resultados & Periódico/Ano \\
\hline $\begin{array}{l}\text { Indicadores de } \\
\text { qualidade utilizados } \\
\text { nos serviços de } \\
\text { enfermagem de } \\
\text { hospitais de ensino. }{ }^{12}\end{array}$ & $\begin{array}{l}\text { Apresentar aspectos } \\
\text { cruciais de engajamento; } \\
\text { Promover ambiente de } \\
\text { satisfação e respeito; }\end{array}$ & $\begin{array}{l}\text { Melhoria da experiência dos } \\
\text { pacientes. Adaptação no setor de } \\
\text { emergência visando melhorar a } \\
\text { qualidade. Benefícios nos } \\
\text { indicadores de qualidade. }\end{array}$ & $\begin{array}{l}\text { Revisa } \\
\text { Eletrônica de } \\
\text { Enfermagem, } \\
2014\end{array}$ \\
\hline $\begin{array}{c}\text { Protocolo de } \\
\text { avaliação e } \\
\text { classificação de risco } \\
\text { de pacientes em } \\
\text { unidade de } \\
\text { emergência. }{ }^{13} \\
\end{array}$ & $\begin{array}{c}\text { Investigar o uso de } \\
\text { arredondamento } \\
\text { intencional na emergência; } \\
\text { Classificar e reclassificar } \\
\text { os pacientes. }\end{array}$ & $\begin{array}{l}\text { Diminuição do tempo de espera na } \\
\text { sala de emergência e redução dos } \\
\text { riscos associados à demora de } \\
\text { atendimento. }\end{array}$ & $\begin{array}{l}\text { Revista Latino- } \\
\text { Americana de } \\
\text { Enfermagem, } \\
2014\end{array}$ \\
\hline $\begin{array}{l}\text { Perceived } \\
\text { Appropriateness of } \\
\text { Shared Decision- } \\
\text { making in the } \\
\text { Emergency } \\
\text { Departament: a } \\
\text { survey study. }{ }^{14}\end{array}$ & $\begin{array}{c}\text { Descrever percepções e } \\
\text { treinar médicos da } \\
\text { emergência na tomada de } \\
\text { decisões. }\end{array}$ & $\begin{array}{l}\text { As técnicas de arredondamento } \\
\text { intencionais podem levar a } \\
\text { melhorias na segurança do } \\
\text { paciente. } \\
\text { Identificou uma série de } \\
\text { dificuldades e adaptações } \\
\text { necessárias para garantia de } \\
\text { atendimento de paciente agudo. }\end{array}$ & $\begin{array}{c}\text { Academic } \\
\text { Emergency } \\
\text { Medicine, } 2016\end{array}$ \\
\hline $\begin{array}{l}\text { Impact of a Chronic } \\
\text { Pain Protocol on } \\
\text { Emergency } \\
\text { Department } \\
\text { Utilization. }^{15}\end{array}$ & $\begin{array}{l}\text { Gerenciamento dos } \\
\text { pacientes com dor crônica } \\
\text { no serviço de emergência. }\end{array}$ & $\begin{array}{l}\text { Reduções do número de visitas de } \\
\text { retorno no serviço de emergência e } \\
\text { do número de medicamentos } \\
\text { prescritos. }\end{array}$ & $\begin{array}{c}\text { Academic } \\
\text { Emergency } \\
\text { Medicine, } 2016\end{array}$ \\
\hline $\begin{array}{l}\text { The impact of flexible } \\
\text { care area on } \\
\text { throughput measures } \\
\text { inan academic } \\
\text { emergency } \\
\text { Departament. } 16\end{array}$ & $\begin{array}{c}\text { Avaliar a aplicação de } \\
\text { protocolos para reduzir o } \\
\text { tempo de atendimento na } \\
\text { emergência. }\end{array}$ & $\begin{array}{c}\text { Adequado para deliberar sobre os } \\
\text { procedimentos invasivos. } \\
\text { O uso do protocolo reduz o risco } \\
\text { para a equipe e pacientes. }\end{array}$ & $\begin{array}{l}\text { Journal of } \\
\text { Clinical Nursing, } \\
2015\end{array}$ \\
\hline $\begin{array}{l}\text { Communication } \\
\text { between residential } \\
\text { aged care facilities } \\
\text { and the emergency } \\
\text { department: a review } \\
\text { of the literature. }{ }^{7} \\
\end{array}$ & $\begin{array}{l}\text { Explorar as práticas de } \\
\text { comunicação na gestão do } \\
\text { cuidado da equipe de } \\
\text { residentes na emergência. }\end{array}$ & $\begin{array}{l}\text { A equipe utiliza informações clínicas } \\
\text { e pessoais para planejar uma } \\
\text { assistência de qualidade. }\end{array}$ & $\begin{array}{l}\text { International } \\
\text { Journal of } \\
\text { Nursing Studies. } \\
2014\end{array}$ \\
\hline $\begin{array}{l}\text { Qualidade dos } \\
\text { prontos-socorros e } \\
\text { prontos- } \\
\text { atendimentos: a } \\
\text { satisfação dos } \\
\text { usuários. }{ }^{18}\end{array}$ & $\begin{array}{l}\text { Avaliar a gestão da } \\
\text { qualidade dos serviços } \\
\text { médicos através da } \\
\text { utilização e distribuição de } \\
\text { recursos. }\end{array}$ & $\begin{array}{l}\text { Melhorar a estrutura física. } \\
\text { Evitar superlotação das } \\
\text { emergências. } \\
\text { Aplicar investimentos nas estruturas } \\
\text { físicas e nas equipes profissionais. }\end{array}$ & Einstein, 2015 \\
\hline $\begin{array}{l}\text { Analysis of the equity } \\
\text { of emergency } \\
\text { medical services: a } \\
\text { cross-sectional } \\
\text { survey in Chongqing } \\
\text { city. }{ }^{19}\end{array}$ & $\begin{array}{l}\text { Avaliação da assistência e } \\
\text { do processo de trabalho. }\end{array}$ & $\begin{array}{l}\text { Reorganização dos fluxos das } \\
\text { emergências. Investir na qualidade } \\
\text { do serviço de emergência. Aplicar } \\
\text { protocolos melhorando } \\
\text { atendimento. }\end{array}$ & $\begin{array}{l}\text { International } \\
\text { Journal for } \\
\text { Equity in Health, } \\
2015\end{array}$ \\
\hline
\end{tabular}


e) desestruturação técnica e operativa das equipes de plantão; f) despreparo no atendimento dos casos emergenciais ${ }^{3,20}$.

$\mathrm{Na}$ segunda característica, é destacada a forma de acolhimento nas recepções dos serviços de emergência, onde funcionários do setor de segurança definem a prioridade do atendimento. Talvez aqui, se identifique o aspecto mais grave do atendimento nesses serviços, quando o indivíduo, para além das péssimas condições do ambiente, fica exposto a um critério espúrio de seleção dos seus problemas, em que os casos graves não são priorizados ${ }^{3,20}$.

$\mathrm{Na}$ revisão da literatura sobre a qualidade dos serviços de emergência no critério dos indicadores, pode-se resgatar 0 pensamento expresso por Arouca: a unidade mais simples de análise a ser considerada, no interior da Medicina, é o cuidado médico, que envolve uma relação entre duas pessoas. Descrita como uma relação que se dá em um espaço especializado para suprir, resolver ou atender a esse conjunto de necessidades denominadas doença, espaço este que é a emergência ${ }^{21}$.

A gestão do cuidado nos serviços de emergência deve ser realizada por uma equipe multiprofissional, formada por médicos, enfermeiros, técnicos de enfermagem, fisioterapeutas, assistentes sociais, psicólogos, nutricionistas e farmacêuticos, integrados em um plano terapêutico individualizado, com problemas, metas e responsáveis devidamente registrados e acompanhado em um prontuário único. Tendo como resultados a ampliação das responsabilidades dos atendimentos no serviço hospitalar de emergência e dos processos de trabalho 22,23 .

\section{Conclusão}

A maioria dos estudos desta revisão destacou a avaliação dos serviços de saúde no critério qualidade da assistência, correspondendo a quatro estudos do total encontrado. Os demais estudos apresentaram quantitativos bem semelhantes, enfocando a qualidade em relação a estrutura física, enfatizando o processo de trabalho, a capacitação dos profissionais e a resolutividade do setor de emergência que impacta no funcionamento do hospital como um todo. Diante do pequeno número de publicações encontradas sobre 0 tema, podemos inferir a relevância de amis estudos detalhando a dinâmica gerencial no setor da emergência.

O desafio dos gestores na atual situação dos serviços de emergência constitui-se em avançar na estruturação de serviços que contemplem a regulação dos atendimentos, as redes de referência, a constituição do comitê gestor de emergência, a capacitação dos profissionais e ambiente físico estruturado ao atendimento das emergências, priorizando a vida do usuário do sistema de saúde. 


\section{Referências}

1. Barbosa LR, Melo MRAC. Relações entre qualidade da assistência de enfermagem: revisão integrativa da literatura. Rev Bras Enferm. 2008;61(3):366-70.

2. Brasil. Ministério da Saúde. Portaria no 2.395, de 11 de outubro de 2011. Organiza o Componente Hospitalar da Rede de Atenção às Urgências no âmbito do Sistema Único de Saúde (SUS). Diário Oficial da Republica Federativa do Brasil, Brasília (DF); 2011 out 13; Seção 1:79-82.

3. Domiciano V, Fonseca AS. Tempo médio para atendimento do cliente em um departamento de emergência de um hospital privado. Nursing. 2008;11(119):182-8.

4. Kurcgant $P$, Tronchin DMR, Melleiro MM. A construção de indicadores de qualidade para a avaliação de recursos humanos nos serviços de enfermagem: pressupostos teóricos. Acta Paul Enferm. 2006;19(1):88-91.

5. Oliveira GN, Silva MFN, Araujo IEM, Carvalho Filho MA. Perfil da população atendida em uma unidade de emergência referenciada. Rev LatinoAm Enfermagem. 2011;19(3):1-9.

6. D'Innocenzo M, Adami NP, Cunha ICKO. O movimento pela qualidade nos serviços de saúde e enfermagem. Rev Bras Enferm. 2006;59(1):84-8.

7. Donabedian A. Criteria, norms and standarts of quality: what do they mean. Am J Public Health. 1981 April; 71(4): 409-12.

8. Poll MA, Lunardi VL, Lunardi Filho WD. Atendimento em unidade de emergência: organização e implicações éticas. Acta Paul Enferm. 2008;21(3):509-14.

9. Instituto Brasileiro de Geografia e Estatística. Município de São Luís. Rio de Janeiro: IBGE; 2015 [acesso em 2017 nov 22]. Disponível em: http://www.cidades.ibge.gov.br/xtras/p erfil.php?lang $=\&$ codmun $=211130 \&$ sear $\mathrm{ch}=$ maranhao|sao-luis.

10. Almeida HMS. Programa de Qualidade do Governo Federal aplicado à Saúde. Rev Adm Saúde. 2001;3(12):5-10.

11. Balsanelli AP, Jericó MC. Os reflexos da gestão pela qualidade total em instituições hospitalares brasileiras. Acta Paul Enferm. 2005;18(4):397402.

12. Rossaneis $M A<$ Gabriel CS, Haddad MCFL, Melo MRAC, Bernardes A. Indicadores de qualidade utilizados nos serviços de enfermagem de hospitais de ensino. Rev Eletr Enf. 2014;16(4):769-76.

13. Silva MFN, Oliveira GN, PergolaMarconato AM, Marconato RS, Bargas EB, Araujo IEM. Protocolo de avaliação e classificação de risco de pacientes em unidade de emergência. Rev Latino-Am Enfermagem. 2014;22(2):255-61.

14. Probst MA, Kanzaria HK, Frosch DL, Hess EP, Winkel G, Ngai KM, et al. Perceived appropriateness of shared decision-making in the emergency department: a survey study. Acad Emerg Med. 2016;23(4):375-81.

15. Olsen JC, Ogarek JL, Goldenberg EJ, Sulo S. Impact of a chronic pain protocol on emergency department utilization. Acad Emerg Med. 2016;23(4):424-32.

16. McGrath J, LeGare A, Hermanson L, Repplinger MD. The impact of a flexible care area on throughput measures in an academic emergency department. J Emerg Nurs. 2015;41(6):503-9.

17. Griffiths D, Morphet J, Innes K, Crawford K, Williams A. Communication between residential aged care facilities and the emergency department: a review of the literature. Int J Nurs Stud. 2014;51(11):1517-23.

18. Lima CA, Santos BTP, Andrade DLB, Barbosa FA, Costa FM, Carneiro JA. Qualidade dos prontos-socorros e 
prontos-atendimentos: a satisfação dos usuários. Einstein. 2015;13(4):587-93.

19. Liu Y, Jiang $Y$, Tang S, Quu J, Zhong $X$, Wang $Y$. Analysis of the equity of emergency medical services: a crosssectional survey in Chongqing city. Int J Equity Health. 2015;14:150.

20. Deslandes SF. Frágeis Deuses: profissionais da emergência entre danos da violência e recriação da vida. Rio de Janeiro: Fiocruz; 2002.

21. Arouca ASS. Medicina preventiva e sociedade. In: O dilema preventivista: contribuição para a compreensão e crítica da medicina preventiva. Rio de Janeiro: UNESP/ FIOCRUZ; 2003.

22. Merhy EE. Saúde: a cartografia do trabalho vivo. São Paulo: Hucitec; 2002.

23. Rollo AA. É possível construir novas práticas assistenciais no hospital público? In: Merhy EE, Onocko R. Agir em saúde: um desafio para o público. São Paulo: Hucitec; 1997. p. 321-39. 